\title{
Effects of Xinfeng Capsules on Expression of Platelet Granule Membrane Protein 140 and Platelet Cluster of Differentiation 40 Ligand in Peripheral Blood of Adjuvant Arthritis Rats
}

\author{
Zong Rui-kai ${ }^{1}$ and Liu Jian ${ }^{2}$ \\ ${ }^{1}$ Hubei University of Traditional Chinese Medicine, Hubei, Wuhan 430065, China \\ ${ }^{2}$ Department of Rheumatism and Immunity, The First Hospital Affiliated to Anhui College of Traditional Chinese Medicine, \\ Anhui, Hefei 230031, China
}

Correspondence should be addressed to Liu Jian, liujianahzy@yahoo.com.cn

Received 25 December 2011; Accepted 6 February 2012

Academic Editor: Ruben Burgos-Vargas

Copyright ( 2012 Z. Rui-kai and L. Jian. This is an open access article distributed under the Creative Commons Attribution License, which permits unrestricted use, distribution, and reproduction in any medium, provided the original work is properly cited.

Platelet GMP-140 and CD40L as specific markers of platelet activation play an important role in the morbidity and development of rheumatoid arthritis. The expression of GMP-140, CD40L increases in peripheral blood of AA rats. And they have correlation with voix pedis' swelling, AI. XFC could inhibit the inflammatory response through inhibiting platelet activation of AA rats, which means decreasing the expression of GMP-140, CD40L in peripheral blood. So, the voix pedis' swelling and AI were decreased as the result.

\section{Introduction}

Studies show that platelet activation plays an important role in the pathogenesis of rheumatoid arthritis [1-3]. Activation of platelet releases and expresses a variety of activation products to the membrane surface. These substances participate in platelet-endothelial cell reaction and plateletleukocyte reaction in different ways and play an important role in the occurrence and development of inflammation and particularly, chronic inflammatory [4]. To explore the mechanism of platelet activation in the pathogenesis of rheumatoid arthritis, the rats were injected with Freund's complete adjuvant (FCA) as immune stimulation, to observe the change of platelet GMP-140 and CD40L in peripheral blood and effects of Xinfeng Capsules (XFC) on them. The result of study is as follows.

\section{Material and Methods}

2.1. Animals. 40 male clean degree SD rats, Anli laboratory animals limited liability company of Anhui Province (certificate number: $2010-0008)$, average weight $(200 \pm 20) \mathrm{g}$.
2.2. Major Drugs and Reagents. Methotrexate (MTX): $2.5 \mathrm{mg}$ per tablet, Sine Pharmaceutical factory of Shanghai pharmaceutical limited, batch number: 100923; tripterygium wilfordii polycoride Tablet (TPT): $10 \mathrm{mg}$ per tablet, Shanghai Fudan Fuhua pharmaceutical industry limited, batch number: 100809. XFC: preparation centre of first hospital affiliated to Anhui College of Traditional Chinese Medicine, main composition: coix seed, Astragalus, centipede, tripterygium wilfordii, $0.4 \mathrm{~g}$ per pill, batch number: 100521; FCA: USA Sigma company, batch number: 065K7805; anti-rat GMP140 and CD40L monoclonal antibody labeled with Fluorescein Isothiocyanate (FITC): Beijing Bioss biotechnology Co. Ltd, batch number: 100512 and 100419, Art. No. bs-1286R and bs-0561R.

2.3. Major Instruments and Equipments. Beckman-Coulter XL-ESPIC MCL-type Flow Cytometry.

2.4. Model and Administration. $40 \mathrm{SD}$ male rats were randomly divided into 5 groups after adaptable feeding 1 week, normal control group $(n=8)$, and model groups $(n=32)$. Except for the rats of normal group, the others were 
intracutaneously injected with $0.1 \mathrm{~mL}$ of Freund's complete adjuvant in the right hindlimb to model [5]. AA rats were randomly divided into 4 groups and administrated at 20 times clinical dosage: model control group, MTX group, TPT group, and XFC group after 19th day. (1) TPT group: TPT was grinded into fine powder and mixed with saline to blending. The rats were intragastric administrated with $10 \mathrm{~mL} / \mathrm{kg} 1$ time each day. (2) MTX group: MTX was grinded into fine powder and mixed with saline to blending. The rats were intragastric administrated with $10 \mathrm{~mL} / \mathrm{kg} 1$ times a week, while they were given saline $10 \mathrm{~mL} / \mathrm{kg} 1$ times each day on the other days. (3) XFC group: XFC was grinded into fine powder without capsule and mixed with saline to blending. The rats were intragastric administrated with $10 \mathrm{~mL} / \mathrm{kg} 1$ times a day. (4) Normal control group and model control group were given saline $10 \mathrm{~mL} / \mathrm{kg} 1$ times each day. The rats of each group were intragastric administrated for $30 \mathrm{~d}$.

2.5. The Voix Pedis' Swelling. Left voix pedis volumes of rats were measured on $1 \mathrm{~d}$ before injection, $1 \mathrm{~d}$ before administration, and $30 \mathrm{~d}$ after administration. The voix pedis' swelling was calculated [6]. The voix pedis' swelling = $(V t-V n) / V n \times 100 \%(V n$, before injection; $V t$, after injection).

2.6. Arthritis Index (AI). Observe and record degree of arthropathy on 12th, 1 time three day. The arthropathy was appraised with 5-point method. AI was calculated according to the other three limbs without injection [7]. 0 score: no redness and swelling; 1 score: redness and swelling of little toe joint; 2 score: swelling of toe and voix pedis joint; 3 score: swelling of joints under ankle joint; 4 score: swelling of all joints includes ankle joint; 5 score: integral accumulation of each joint is AI of rats.

2.7. GMP-140. Blood was drawn to EDTA anticoagulant tube and then centrifuged for 8 minutes at $1000 \mathrm{r} / \mathrm{min}$. Platelet rich plasma (PRP) is plasma without liquid supernatant. Then adding anti-rat GMP-140 monoclonal antibody labeled with FITC to it. And incubating for 15 minutes in dark at room temperature. Adding $2 \mathrm{~mL} \mathrm{1 \%}$ paraformaldehyde fixative and putting it to flow cytometry. Last, analyzing GMP-140 positive expression with CeQuest.

2.8. CD40L. Adding anti-rat CD40L monoclonal antibody labeled with FITC to $10 \mu \mathrm{L}$ blood with EDTA anticoagulant. And incubating for 5 minutes in dark at room temperature. Then centrifuging for 5 minutes at $1000 \mathrm{r} / \mathrm{min}$. And throwing away liquid supernatant. Adding phosphate buffer (PBS) $2 \mathrm{~mL}$ and centrifuging for 5 minutes at $1000 \mathrm{r} / \mathrm{min}$. Throwing away liquid supernatant. Mixing with PBS $300 \mu \mathrm{L}$ to blending. Putting it to flow cytometry and analyzing CD40L positive expression with CeQuest.

2.9. Statistics. Data were expressed as $(\bar{x} \pm s)$ and to be treated statistically with SPSS 11.5 for windows software. After Kolmogorov-Smirnov Region Surrounding the Nansha
Islands, multiple groups means were compared with singlefactor analysis of variance, and the comparison among groups was performed with LSD and SNK method. And the samples were analyzed using the method of multivariate correlation analysis.

\section{Result}

3.1. The Change of Weight of Each Group. The weight of each group was no significant difference before phlegmasia $(P>0.05)$. The weight of model group was significantly decreased than the normal group on 1 day before administration $(P<0.05)$. Compared with the model group, the weight of MTX, TPT, and XFC groups was significantly increased $(P<0.01)$. The body mass in XFC-treated group was significantly higher than those in MTX and TPT groups $(P<0.05)$ (see Table 1$)$.

3.2. The Change of the Voix Pedis' Swelling and AI of Each Group. The voix pedis' swelling and AI of the model group was significantly increased than the normal group on 1 day before administration $(P<0.01)$. Compared with the model group, there was no significant difference in three treated groups $(P>0.05)$. After the 30th of administration, the voix pedis' swelling and AI of MTX, TPT, and XFC groups was significantly decreased than those in the model group $(P<0.01)$. There was no significant difference among three treated groups $(P>0.05)$ (see Table 2$)$.

3.3. The Change of GMP-140, CD40L of Each Group. The GMP-140, CD40L, of the model group was significantly higher than the normal group $(P<0.01)$. Compared with the model group, the GMP-140 and CD40L of MTX, TPT and XFC groups were significantly decreased $(P<0.01)$. The GMP-140 of XFC showed a decreasing tendency with no significant difference than the MTX, TPT groups $(P>0.05)$. The CD40L showed no significant difference among three treated groups $(P>0.05)$ (see Table 3 and Figures 1 and 2).

3.4. The Dependability between GMP-140, CD40L, and the Voix Pedis' Swelling, AI of Rats. The expression of GMP140 and CD40L were positively correlated with voix pedis' swelling and AI $(P<0.01$ or $P<0.05)$ (see Table 4$)$.

\section{Discussion}

Platelet GMP-140 and CD40L as specific markers of platelet activation play an important role in the morbidity and development of rheumatoid arthritis [8]. Studies show that GMP-140 was the marker of RA activity and inflammatory $[9,10]$. Activated GMP-140 interacted with polymorphonuclear leukocyte ligand in circulation. Then inflammatory cells were exuded to joint synovial tissues space and produced immune response and inflammatory damage of synovium. Inflammatory cells could release more GMP-140, thus, in cycles, promoting the progression of RA and synovial inflammatory [11]. GMP-140 could activate synovial blood coagulation system. Fibrin degradation products were 


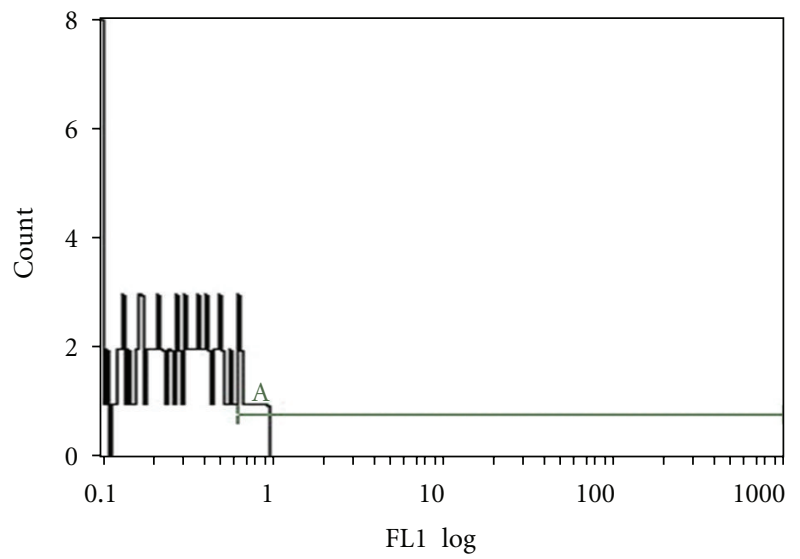

(a) Control

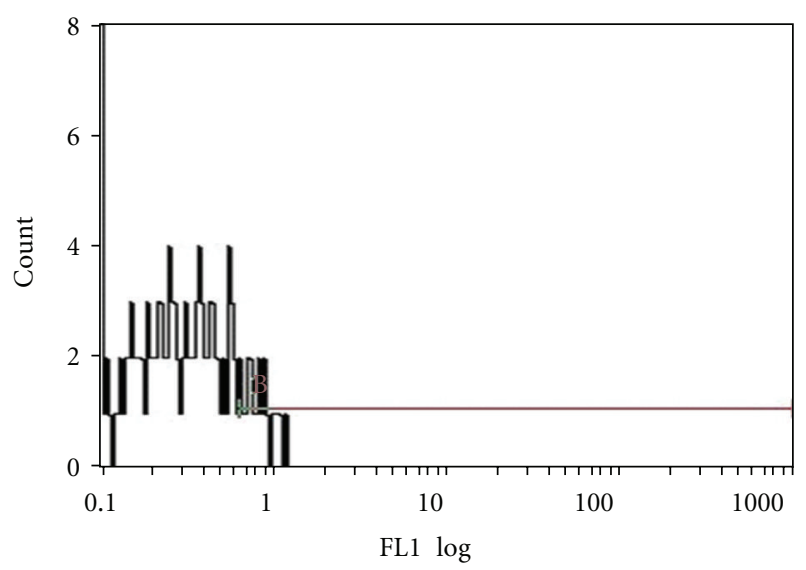

(c) MTX

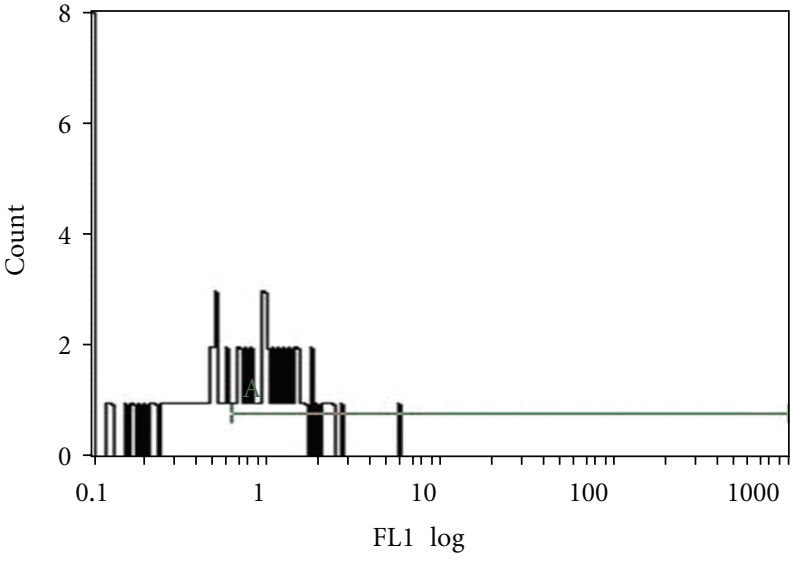

(b) Model

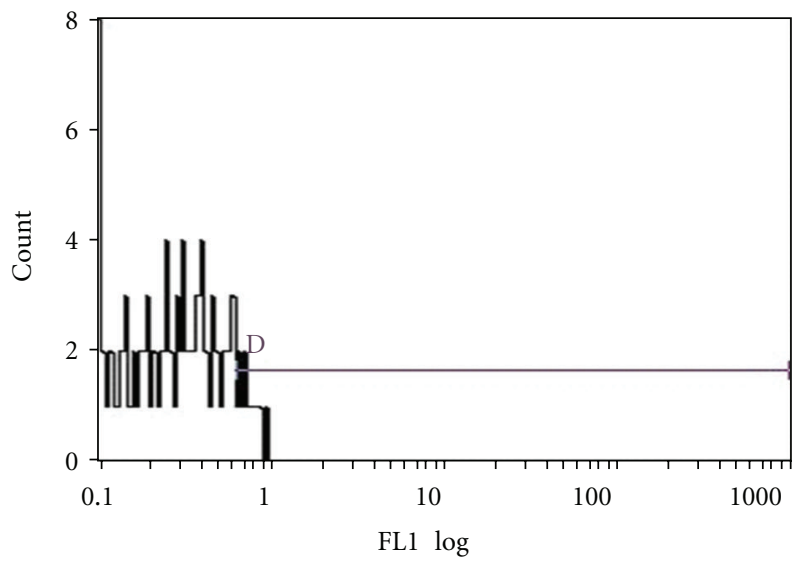

(d) TPT

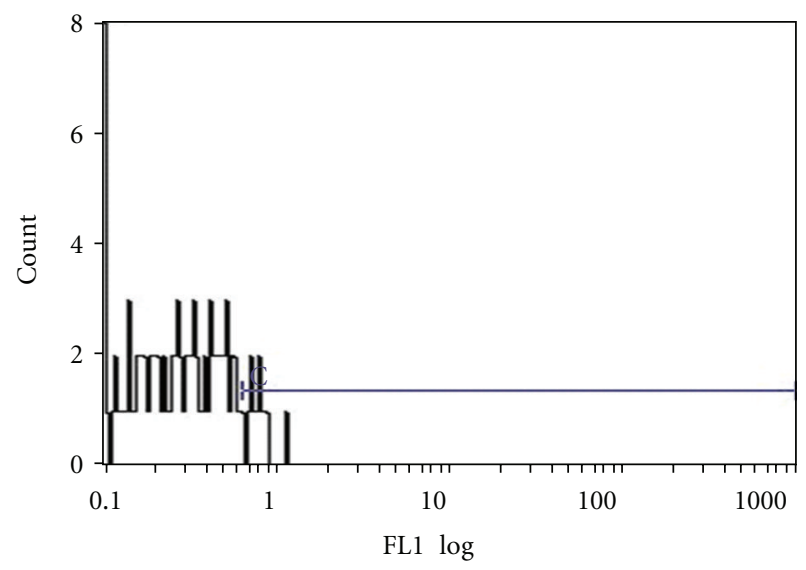

(e) XFC

Figure 1: GMP-140 of AA rats.

deposited in RA synovium. It caused joint swelling and tissue injury [12]. CD40L plays an important role in the occurrence and development of chronic synovitis. High level expression of CD40L has been detected in RA synovium [13]. Expression of CD40L on platelet can induce endothelial cells to release chemotaxins and express adhesion molecules. Thus producing a signal, which raises various inflammatory mediators gathering in synovium. It causes hyperplasia of synovial tissue, inflammation, damage of cartilage and bone [14]. CD40L can also stimulate inflammation mononuclear macrophages, which secrete vascular endothelial growth factor, increase vascular permeability, improve inflammatory infiltration, and promote the growth and relocation of endothelial cells, angiogenesis, and pannus formation [15]. In this experiment, it was shown that GMP-140 and CD40L as platelet activation markers in peripheral blood of AA rats 
TABLE 1: Comparison of weight of each group $(n=8, \bar{x} \pm s, \mathrm{~g})$.

\begin{tabular}{|c|c|c|c|}
\hline \multirow{2}{*}{ Groups } & \multicolumn{3}{|c|}{ Weight } \\
\hline & Weight before phlegmasia & Weight before administration & Weight of the 30th of administration \\
\hline Control & $214.38 \pm 18.98$ & $260.00 \pm 11.02$ & $360.25 \pm 23.46$ \\
\hline Model & $211.88 \pm 17.31$ & $226.25 \pm 35.73^{*}$ & $260.50 \pm 37.20^{* *}$ \\
\hline MTX & $213.75 \pm 13.02$ & $231.88 \pm 30.70^{*}$ & $311.00 \pm 43.61^{* \Delta \Delta}$ \\
\hline $\mathrm{TPT}$ & $212.50 \pm 11.95$ & $230.00 \pm 14.88^{*}$ & $317.63 \pm 32.51^{* \Delta \Delta}$ \\
\hline $\mathrm{XFC}$ & $213.75 \pm 22.80$ & $232.50 \pm 13.63^{*}$ & $351.88 \pm 21.96^{\Delta \Delta \Delta \square}$ \\
\hline
\end{tabular}

Note: compared with control group, ${ }^{*} P<0.05,{ }^{* *} P<0.01$; compared with model group, $\triangle \triangle \mathbf{\Delta} P<0.01$; compared with MTX group, $\Delta P<0.05$; compared with TPT group, $\square_{P}<0.05$.

TABLE 2: Comparison of the voix pedis' swelling, arthritis index of each group $(n=8, \bar{x} \pm s)$.

\begin{tabular}{|c|c|c|c|c|}
\hline \multirow{2}{*}{ Groups } & \multicolumn{2}{|c|}{ The voix pedis' swelling } & \multicolumn{2}{|c|}{ AI } \\
\hline & $\begin{array}{l}\text { Weight before } \\
\text { administration }\end{array}$ & $\begin{array}{l}\text { Weight of the 30th of } \\
\text { administration }\end{array}$ & $\begin{array}{l}\text { Weight before } \\
\text { administration }\end{array}$ & $\begin{array}{c}\text { Weight of the 30th of } \\
\text { administration }\end{array}$ \\
\hline Control & $33.28 \pm 10.90$ & $38.94 \pm 24.69$ & $0.00 \pm 0.00$ & $0.00 \pm 0.00$ \\
\hline Model & $65.99 \pm 16.49^{* *}$ & $79.20 \pm 24.59^{* *}$ & $7.63 \pm 0.92^{* *}$ & $8.13 \pm 0.64^{* *}$ \\
\hline MTX & $65.09 \pm 14.11^{* *}$ & $46.00 \pm 14.94^{\Delta \Delta}$ & $7.25 \pm 1.04^{* *}$ & $4.00 \pm 0.93^{* * \Delta \Delta}$ \\
\hline TPT & $62.59 \pm 20.19^{* *}$ & $44.92 \pm 20.37^{\Delta \Delta}$ & $7.13 \pm 1.13^{* *}$ & $3.88 \pm 1.25^{* * \Delta \Delta}$ \\
\hline $\mathrm{XFC}$ & $68.86 \pm 16.41^{* *}$ & $41.57 \pm 15.28^{\Delta \Delta}$ & $7.88 \pm 0.64^{* *}$ & $3.50 \pm 1.07^{* * \Delta \Delta}$ \\
\hline
\end{tabular}

Note: compared with control group, ${ }^{* *} P<0.01$; compared with model group, ${ }^{\triangle \triangle} P<0.01$.

TABle 3: Comparison of GMP-140, CD40L of each group $(n=8$, $\bar{x} \pm s, \mathrm{pg} / \mathrm{mL})$.

\begin{tabular}{lcc}
\hline Groups & GMP-140 & CD40L \\
\hline Control & $0.30 \pm 0.17^{\Delta \Delta}$ & $94.71 \pm 2.42^{\Delta}$ \\
Model & $1.07 \pm 0.52^{* *}$ & $98.16 \pm 1.39^{* *}$ \\
MTX & $0.55 \pm 0.27^{\Delta \Delta}$ & $95.47 \pm 2.36^{\Delta}$ \\
TPT & $0.49 \pm 0.28^{\Delta \Delta}$ & $95.24 \pm 1.90^{\Delta}$ \\
XFC & $0.38 \pm 0.20^{\Delta \Delta}$ & $95.39 \pm 2.27^{\Delta}$ \\
\hline
\end{tabular}

Note: compared with control group, ${ }^{* *} P<0.01$; compared with model group, ${ }^{\triangle} \mathrm{P}<0.01,{ }^{\triangle} P<0.05$.

TABle 4: The dependability between GMP-140, CD40L, and the voix pedis' swelling, AI $(n=40)$.

\begin{tabular}{lcccc}
\hline & \multicolumn{2}{c}{ GMP-140 } & \multicolumn{2}{c}{ CD40L } \\
& $r$ & $P$ & $r$ & $P$ \\
\hline The voix pedis' swelling & $.608^{* *}$ & .000 & $.441^{*}$ & .015 \\
AI & $.611^{* *}$ & .000 & $.386^{*}$ & .035 \\
\hline$* * P<0.01,{ }^{*} P<0.05$. & & & &
\end{tabular}

were significantly increased. The reason was that activated platelet in peripheral blood of AA rats releases a large number of activation products including platelet CD40L, GMP-140. This study also discovered that GMP-140 and CD40L were positively correlated with voix pedis' swelling and AI $(P<$ 0.01 or $P<0.05$ ). GMP-140 and CD40L as proinflammatory mediators promote the occurrence and development of joint synovitis and pannus formation. So, platelet GMP-140 and CD40L have relevance with inflammatory markers.
XFC, which strengthens the spleen and replenishes Qi, resolving Dampness and freeing channel, is a traditional Chinese medicine compound preparation based on overall regulation. Its main components are coix seed, astragalus, tripterygium wilfordii, and centipede. They not only have different degrees of anti-inflammatory effect but also inhibit platelet activation. Astragalus has obvious anti-inflammatory effects. Its mechanism could lie in decreasing the emergence of oxyradical, inflammatory mediators including IL-8, TNF$\alpha, \mathrm{NO}$, and so on. And adding the expression of glucocorticoid receptor under inflammation $[16,17]$, astragalus can effectively reduce the degree of platelet activation and inhibit synthesis of platelet GMP-140. Modern study confirmed that it could obviously inhibit joint swelling induced by egg white, granuloma induced by cotton ball, and the ear edema in mice induced by xylene. And it also could inhibit inflammatory with inhibiting significantly the synthesis and release of prostaglandin E2 of inflammation [18]. Modern pharmacological studies show that tripterygium wilfordii had a strong anti-inflammation effect with inhibiting the expression of IL- $1 \beta$, IL-12, TNF- $\alpha$, and secretion of NO and IL- 6 from macrophage [19-22]. Adjuvant arthritis rat blood was in hyperviscosity and platelet hyperaggregation. Tripterygium wilfordii could improve blood hyperviscosity and platelet aggregation of AA rat, which assisted anti-inflammatory effect. The research shows that tripterygium wilfordii could suppress immune by inhibiting expression of CD40L [23]. Centipede plays an anti-inflammatory effect with improving microcirculation, extending coagulation time, decreasing hematocrit, reducing blood viscosity, increasing the number of opening microvascular, and increasing diameter of microvascular. 


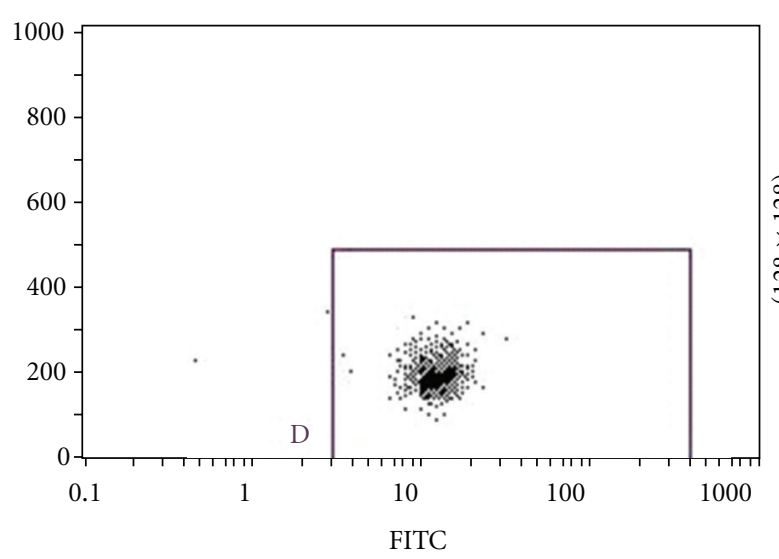

(a) Control

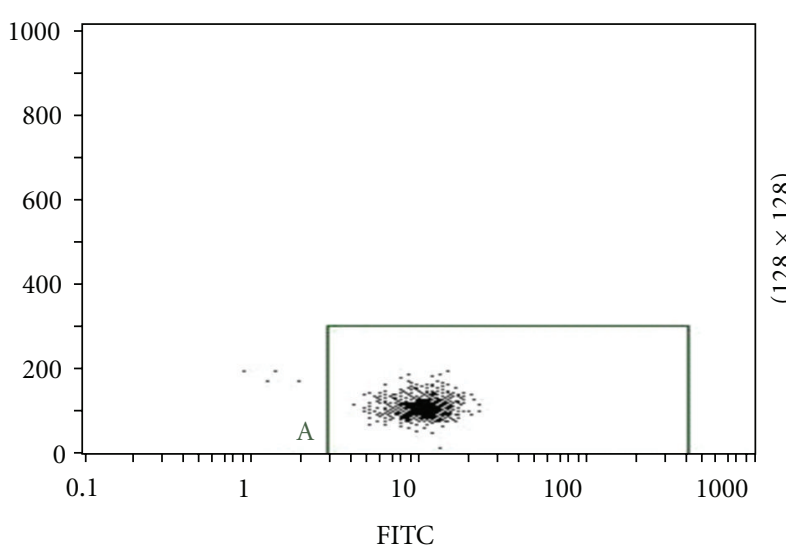

(c) MTX

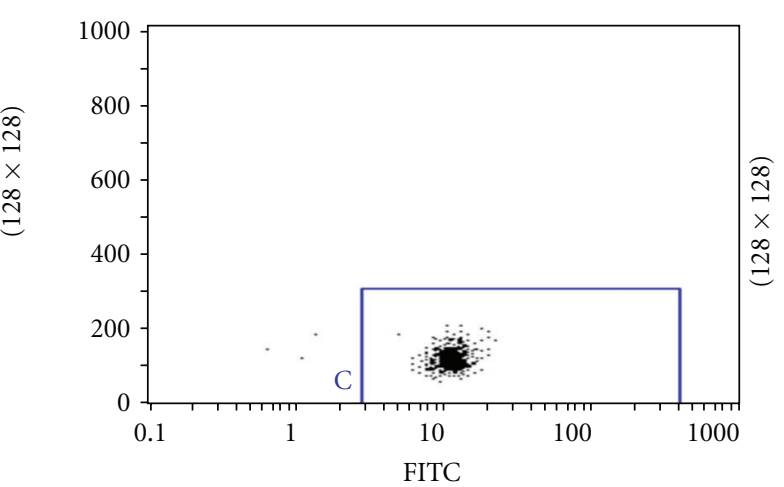

(b) Model

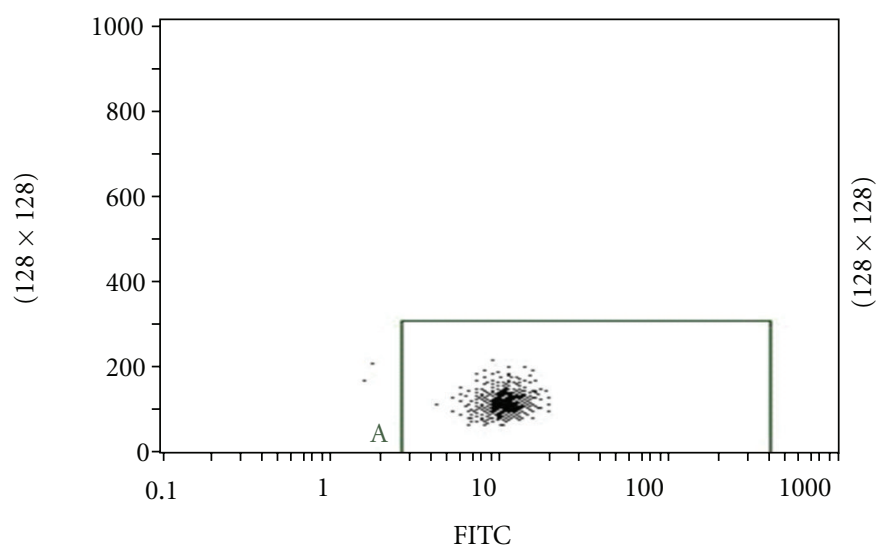

(d) TPT

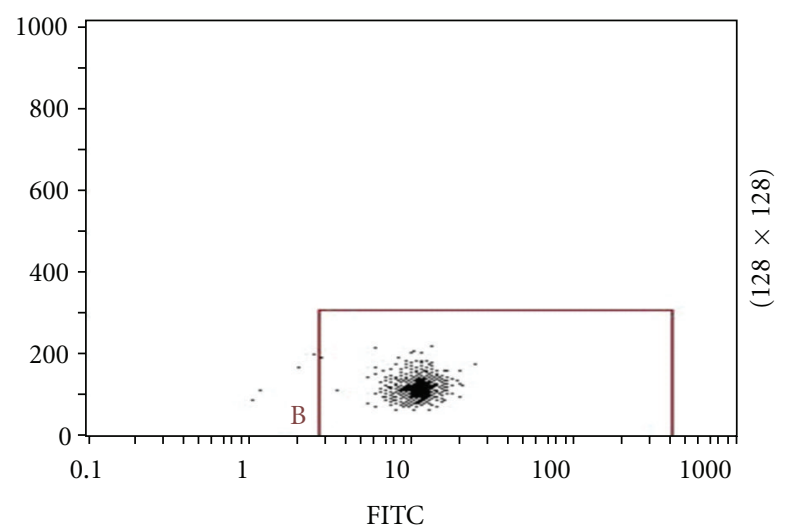

(e) XFC

FIGURE 2: CD40L of AA rats.

The result from the experiment shows that XFC could decrease the voix pedis' swelling and AI of AA rats the same as MTX, TPT. The body mass in XFC-treated group was significantly higher than those in MTX and TPT groups because that astragalus in XFC could protect gastric mucosa and inhibit gastrointestinal adverse reactions of tripterygium wilfordii $(P<0.05)$. It shows that XFC has the antiinflammatory effects as MTX, TPT, and is superior to MTX and TPT in improving overall function of AA rat. Content of tripterygium wilfordii in XFC is lower than TPT. It shows that XFC on the overall effect in AA rat is better than a separate application of TPT. And XFC could increase effect and inhibit toxic effect of tripterygium wilfordii. With the expression of GMP-140, CD40L in peripheral blood of AA rats decreased by XFC; the voix pedis' swelling and AI also dropped. It indicated that XFC could inhibit the 
inflammatory response through inhibiting platelet activation of AA rats, which means decreasing the expression of GMP140, CD40L in peripheral blood. So, the voix pedis' swelling and AI were decreased as the result.

\section{Funding}

This paper was funded by the construction projects of Chinese rheumatology, which are state key disciplines in traditional Chinese medicine (2009) no. 30, and Chinese medicine research project of Health Department in Anhui Province no. 2009ZY 05.

\section{References}

[1] F. Wang, N. S. Wang, and C. G. Yan, "Platelet activation in patients with rheumatoid arthritis at active phase," Chinese Journal of Clinical Rehabilitation, vol. 10, no. 40, pp. 84-86, 2006.

[2] J. Mei, "Significance of determination of platelet CD62P in patients with rheumatoid arthritis," Jiangxi Medical Journal, vol. 42, no. 5, pp. 454-455, 2007.

[3] J. Liu, R. K. Zong, X. F. Yu, and Y. Wang, "Effects of Xinfeng Capsule on platelet parameters, P-select, platelet ultrastructure and its therapeutic effect on rheumatoid arthritis patients in active phase," China Journal of Traditional Chinese Medicine and Pharmacy, vol. 23, no. 12, pp. 1090-1094, 2008.

[4] E. Boilard, P. A. Nigrovic, K. Larabee et al., "Platelets amplify inflammation in arthritis via collagen-dependent microparticle production," Science, vol. 327, no. 5965, pp. 580-583, 2010.

[5] F. Feng, Z. J. Ding, and J. Liu, "Experimental study on the model of adjuvant arthritis rats," Tianjin Pharmacy, vol. 16, no. 2, pp. 1-4, 2004.

[6] S. Y. Xu, R. L. Bian, and X. Chen, Experimental Methodology of Pharmacology, People's Medical Publishing House, Beijing, China, 5th edition, 2002.

[7] J. T. Zhang, Modern Experimental Methods in Pharmacology, Peking University Health Science Center and China Peking Union Medical College Press, Beijing, China, 1998.

[8] A. Y. Gasparyan, A. Stavropoulos-Kalinoglou, D. P. Mikhailidis, K. M. J. Douglas, and G. D. Kitas, "Platelet function in rheumatoid arthritis: arthritic and cardiovascular implications," Rheumatology International, vol. 31, no. 2, pp. 153-164, 2010.

[9] G. E. Pamuk, O. Vural, B. Turgut, M. Demir, O. N. Pamuk, and N. Cakir, "Increased platelet activation markers in rheumatoid arthritis: are they related with subclinical atherosclerosis?" Platelets, vol. 19, no. 2, pp. 146-154, 2008.

[10] F. Wang, N. S. Wang, C. G. Yan, J. H. Li, and L. Q. Tang, "The significance of platelet activation in rheumatoid arthritis," Clinical Rheumatology, vol. 26, no. 5, pp. 768-771, 2007.

[11] I. Takeda, S. Kaise, T. Nishimaki, and R. Kasukawa, "Soluble P-selectin in the plasma of patients with connective tissue diseases," International Archives of Allergy and Immunology, vol. 105, no. 2, pp. 128-134, 1994.

[12] "The changes and significance of CD62p, CD41, TNF- $\alpha$ in patients' serum of rheumatoid arthritis," vol. 48, no. 39, pp. 88-89, 2008.

[13] M. Hanyuda, T. Kasama, T. Isozaki et al., "Activated leucocytes express and secrete macrophage inflammatory protein- $1 \alpha$ upon interaction with synovial fibroblasts of rheumatoid arthritis via a $\beta_{2}$-integrin/ICAM-1 mechanism," Rheumatology, vol. 42, no. 11, pp. 1390-1397, 2003.

[14] M. Quinn and D. Fitzgerald, Platelet Function: Assessment, Diagnosis, and Treatment, Humana Press, Totowa, NJ, USA, 2005.

[15] C. Monaco, E. Andreakos, S. Kiriakidis, M. Feldmann, and E. Paleolog, "T-cell-mediated signalling in immune, inflammatory and angiogenic processes: the cascade of events leading to inflammatory diseases," Current Drug Targets, vol. 3, no. 1, pp. 35-42, 2004.

[16] Y. Yang, J. S. Hou, H. J. Chi, C. Y. Fan, and M. Han, "Effects of astragalus on airway inflammation in the rat pulmonary tissue of chronic obstructive pulmonary disease," Shandong Medical Journal, vol. 50, no. 20, pp. 49-51, 2010.

[17] X. J. Ji, A. Li, M. L. Duan, and S. W. Zhang, "The effect of astragaloside IV on NF- $\kappa$ B and glucocorticoid receptor in monocytes in external inflammation state," Chinese Journal of Clinical Medicine, vol. 17, no. 4, pp. 590-593, 2010.

[18] L. Gao, Z. Y. Zhang, L. Zhang, J. Liu, and G. P. Tian, "Experimental research of anti-inflammation and analgesic effect of yiyiren decoction," Journal of Tianjin University of Traditional Chinese Medicine, vol. 24, no. 1, pp. 17-19, 2005.

[19] K. D. Chu, L. D. Chen, F. Ni, C. Q. Zhou, X. Yao, and W. $\mathrm{Xu}$, "The study on pharmacodynamic action research of total alkaloids of tripterygium wilfordii," vol. 27, no. 1, pp. 31-35, 2011.

[20] Z. D. Zhang, F. Zheng, W. Y. Zheng, and C. Jiang, "Effects of tripterygium wilfordii polycoride tableton on cytokines in collagen induced arthritis rats," Strait Pharmaceutical Journal, vol. 22, no. 5, pp. 57-59, 2010.

[21] X. R. Ren, H. C. Fan, J. Z. Wei, N. Ji, L. Y. Liang, and C. G. Ma, "Efects of triptolide on TNF- $\alpha$, IL-12, TGF- $\beta 1$ in spinal cord of EAE mice," China Modern Medicine, vol. 17, no. 1, pp. 5-6, 2010.

[22] F. Yang, X. J. Bai, K. J. Liu, Y. J. Yang, and Y. Zeng, "Effect of triptolide on secretion of inflammatory cellular factor NO and IL- 6 in mice peritoneal macrophage activated by lipopolysaccharide," Herald of Medicine, vol. 29, no. 1, pp. 9$12,2010$.

[23] X. Peng, "Effect of tripterygium wilforddi hook on the expression of CD40L mRNA in peripheral blood mononuclear cells in patients with systemic lupus erythematosus," China Journal of Leprosy and Skin Diseases, vol. 20, no. 6, pp. 550-551, 2004. 


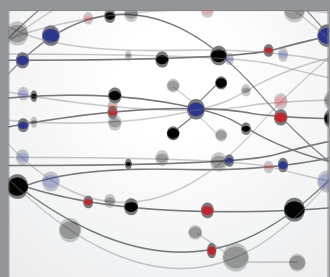

The Scientific World Journal
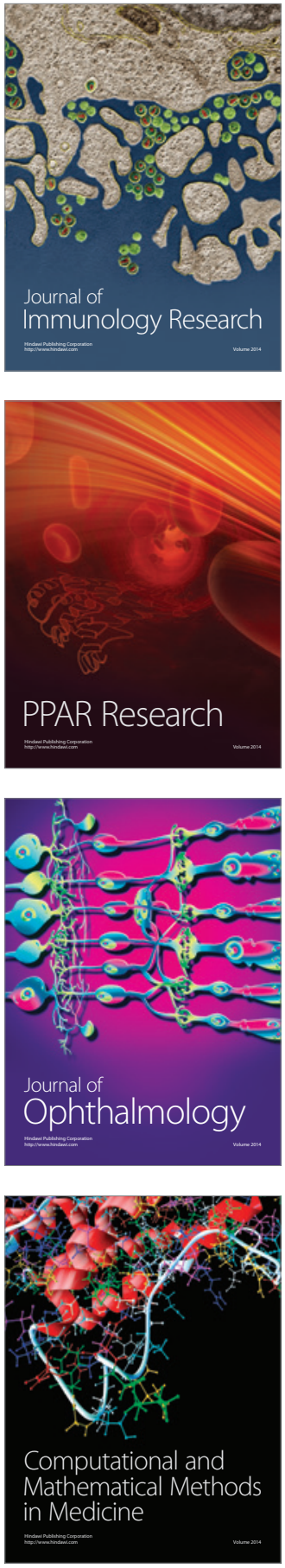

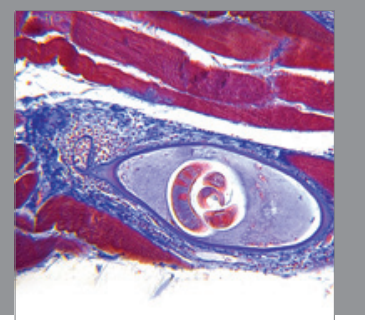

Gastroenterology

Research and Practice
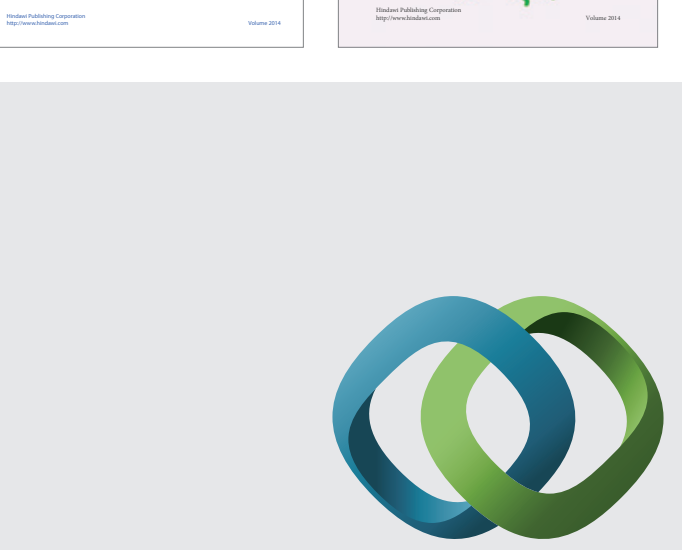

\section{Hindawi}

Submit your manuscripts at

http://www.hindawi.com
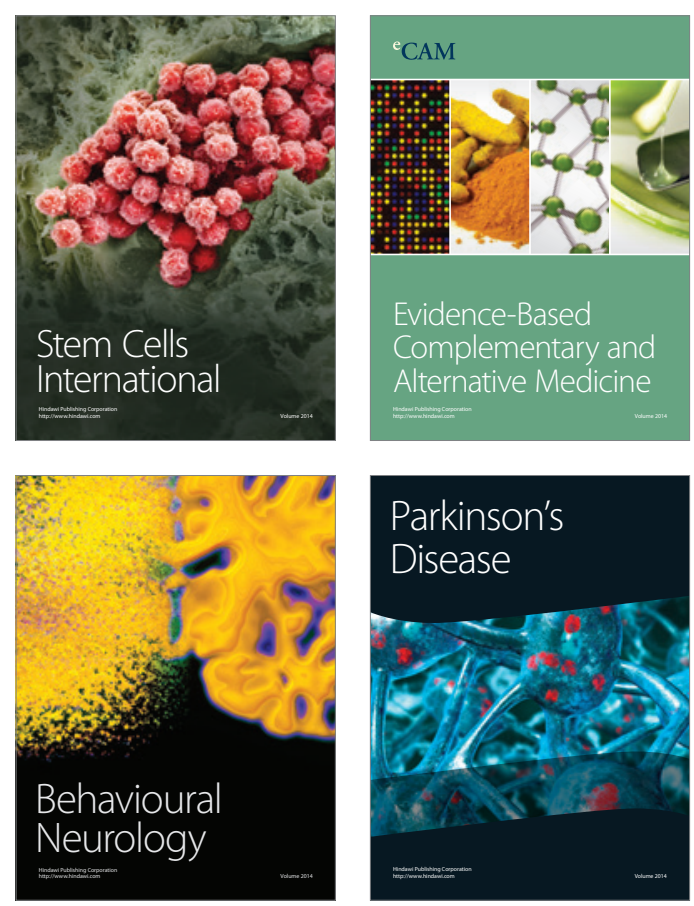

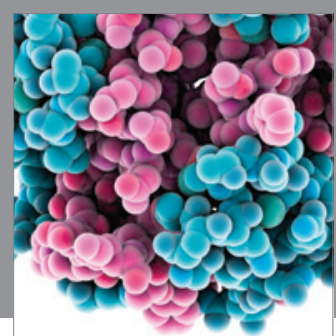

Journal of
Diabetes Research

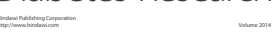

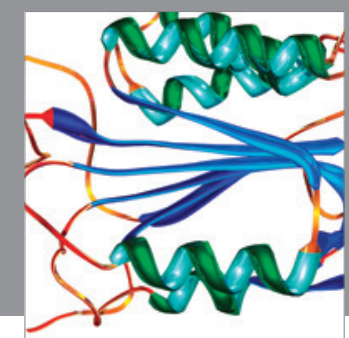

Disease Markers
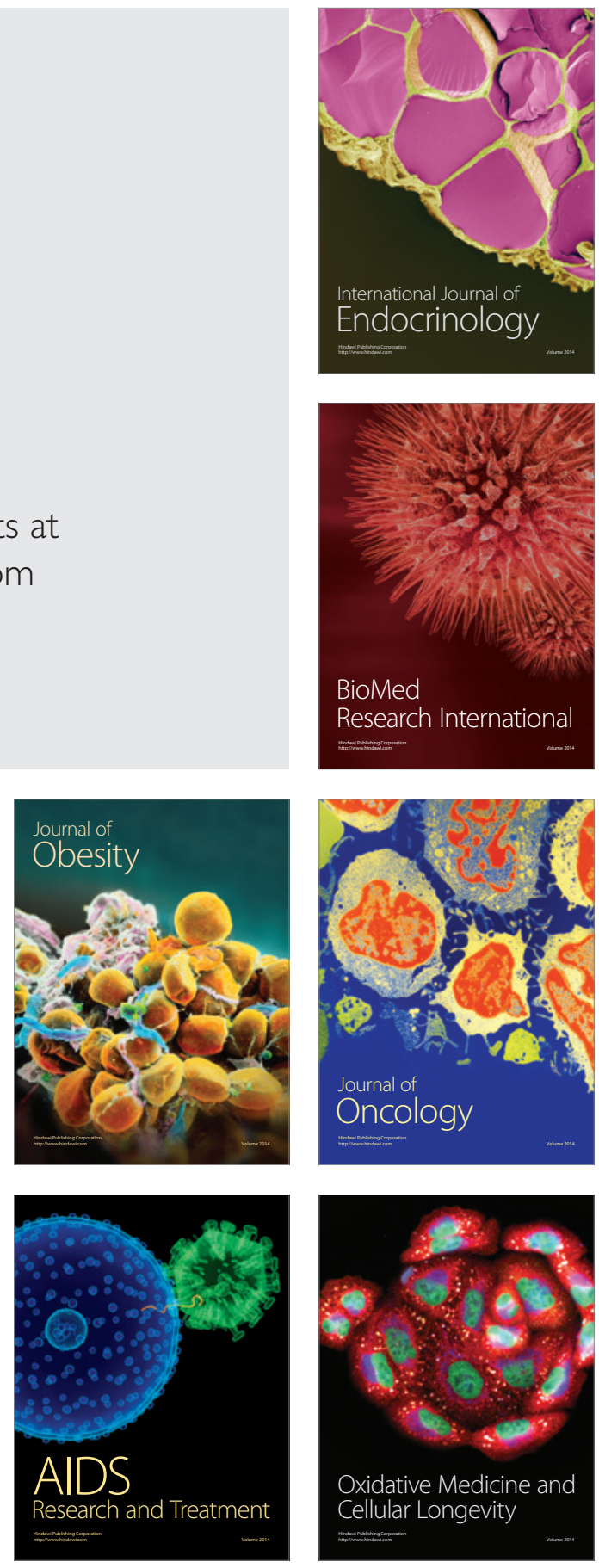